\title{
VLBI observations of $\mathrm{H}_{2} \mathrm{O}$ and $\mathrm{CH}_{3} \mathrm{OH}$ masers in two high-mass YSOs
}

\author{
C. Goddi ${ }^{1}$, L. Moscadelli ${ }^{2}$, A. Sanna ${ }^{3}$, R. Cesaroni ${ }^{2}$ and V. Minier ${ }^{4}$ \\ ${ }^{1}$ Harvard-Smithsonian Center for Astrophysics, 60 Garden St. Cambridge, MA 02138, USA \\ ${ }^{2}$ INAF, Osservatorio Astrofisico di Arcetri, Largo E. Fermi 5, 50125 Firenze, Italy \\ ${ }^{3}$ Dipartimento di Fisica, Universitá degli Studi di Cagliari, S.P. Monserrato-Sestu Km 0.7, \\ 09042 Cagliari, Italy \\ ${ }^{4}$ Service d'Astrophysique, DAPNIA/DSM/CEA Saclay, 91191 Gif-sur-Yvette, France
}

\begin{abstract}
We have conducted phase-reference VLBI observations of $\mathrm{H}_{2} \mathrm{O}$ and $\mathrm{CH}_{3} \mathrm{OH}$ masers toward two high-mass star forming regions, Sh 2-255 IR and AFGL 5142. In Sh 2-255 infrared water masers are aligned along a direction close to the orientation of a large scale $\mathrm{H}_{2}$ jet, tracing possibly shocked material in a precessing jet, or, alternatively, the disk-wind emerging from the disk atmosphere. In AFGL 5142 water masers trace expansion at the base of a protostellar jet, whilst methanol masers are more probably tracing infalling than outflowing gas. Our results suggest that water and methanol masers trace different kinematic structures in the circumstellar gas.
\end{abstract}

\section{Introduction}

Most previous high-angular $\left(\leqslant 0 !^{\prime \prime} 1\right)$ resolution studies of molecular masers in high-mass star forming regions (SFRs) have concentrated mainly on either $\mathrm{H}_{2} \mathrm{O}$ or $\mathrm{CH}_{3} \mathrm{OH}$ masers. While Very Long Baseline Interferometry (VLBI) multi-epoch observations have clarified that water masers originate from shocks associated with protostellar jets (e.g. Goddi et al. 2005), different environments have been proposed in several sources to explain the origin of $\mathrm{CH}_{3} \mathrm{OH}$ masers (Keplerian disks, jets, hot molecular cores, and HII regions). Comparing the spatio-kinematic distribution of both maser species in the same YSO at high-angular resolution better constrains the interpretation of the kinematic structures traced by the masers. So far only a few studies have been performed in both maser types with sufficient angular resolution $(<0$ !' 1$)$ to investigate the nature of the $\mathrm{CH}_{3} \mathrm{OH}$ maser birthplace in SFRs and the association between the $\mathrm{H}_{2} \mathrm{O}$ and $\mathrm{CH}_{3} \mathrm{OH}$ maser emission in the same YSO. Here, we present the results of a multi-epoch VLBI campaign conducted on two high-mass SFRs, Sh 2-255 IR and AFGL 5142, where both the $6.7 \mathrm{GHz}$ methanol and $22.2 \mathrm{GHz}$ water masers are observed.

\section{Observational results}

We performed Very Long Baseline Array (VLBA) multi-epoch observations of the 22.2 GHz water masers and European VLBI Network (EVN) single-epoch observations of the 6.7 GHz methanol masers toward two high-mass YSOs: Sh 2-255 IR and AFGL 5142.

In Sh 2-255 IR, water maser emission consists of three main clusters of features aligned along a direction close to the orientation of a $\mathrm{H}_{2}$ jet (Fig. 1, left panel) observed on angular scales of 1-10 arcsec (Howard et al. 1997). This might suggest that the water maser features are associated with the inner part of the jet. However, all the measured proper motions form large angles with the outflow axis. The observed spatial and velocity distribution of water masers might be explained considering either jet precession or rotation 

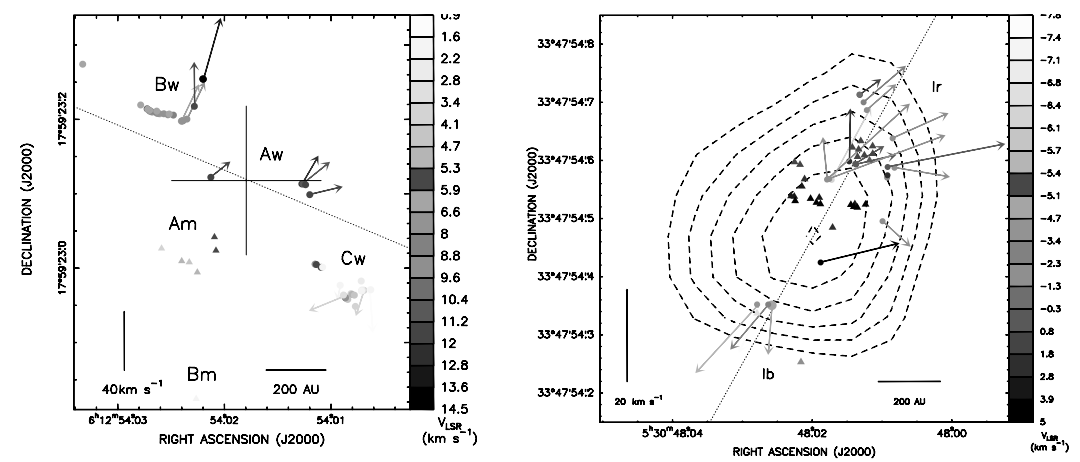

Figure 1. Positions and velocities of VLBA $\mathrm{H}_{2} \mathrm{O}$ (filled circles) and $\mathrm{EVN} \mathrm{CH}_{3} \mathrm{OH}$ (filled triangles) masers, for Sh 2-255 IR (left panel) and AFGL 5142 (right panel), respectively. Different grayscale tones denote the maser line-of-sight velocities and the arrows indicate the measured absolute proper motions of the $\mathrm{H}_{2} \mathrm{O}$ masers. The dotted lines indicate the axes of the $\mathrm{H}_{2}$ jet (Howard et al. 1997) and the CO outflow (Zhang et al. 2007) observed in Sh 2-255 IR and AFGL 5142, respectively. The cross indicates the position uncertainty of the VLA $15 \mathrm{GHz}$ continuum emission peak detected in Sh 2-255 IR (Rengarajan et al. 1996), whereas the contour map shows the $22 \mathrm{GHz}$ VLA continuum emission in AFGL 5142 (Goddi \& Moscadelli 2006).

around the jet axis. The data in hand do not allow us to discriminate unambiguously between the two scenarios. The $6.7 \mathrm{GHz}$ methanol masers were observed with the EVN by Minier et al. (2000). The present data are too scarce even to attempt a qualitative interpretation of their birthplace. New multi-epoch EVN observations of the $6.7 \mathrm{GHz}$ methanol masers are scheduled in order to determine their absolute positions and proper motions.

In AFGL 5142, the water masers show a bipolar spatial and line-of-sight (LOS) velocity distribution; their proper motions clearly indicate that $\mathrm{H}_{2} \mathrm{O}$ masers trace expansion from the YSO along a NW-SE direction (Fig. 1, right panel). Recent SMA observations (Zhang et al. 2007) revealed a $\mathrm{CO}$ outflow oriented along a similar direction, suggesting that water masers might trace the innermost portion of the $\mathrm{CO}$ outflow. The methanol masers, which on the plane of the sky lie closer to the flow center than $\mathrm{H}_{2} \mathrm{O}$ masers, show higher LOS velocities, in disagreement with a Hubble flow (as observed in the CO emission). Since the $6.7 \mathrm{GHz}$ masers are seen in projection against the HII region, which is optically thick at $6.7 \mathrm{GHz}$ (Goddi \& Moscadelli 2006), the $6.7 \mathrm{GHz}$ masers must be located in the foreground of the HII region. The fact that most of the spots present strongly red-shifted l.o.s. velocities suggests that they are tracing gas infalling toward the YSO.

\section{Conclusions}

Our VLBI data reveal a true association of both $\mathrm{H}_{2} \mathrm{O} 22.2 \mathrm{GHz}$ and $\mathrm{CH}_{3} \mathrm{OH} 6.7 \mathrm{GHz}$ masers with the same massive YSO in AFGL 5142. Notwithstanding that, the two species appear to trace different kinematic structures, in particular expansion at the base of a molecular outflow and infall in a molecular envelope, respectively.

\section{References}

Goddi, C., Moscadelli, L., Alef, W., Tarchi, A., \& Pani, M. 2005, A $\& A 432,161$

Goddi, C., \& Moscadelli, L. 2006, A\&A 447, 577

Howard, E. M., Pipher, J. L., \& Forrest, W. J. 1997, ApJ 481, 327

Minier, V., Booth, R. S., \& Conway, J. E. 2000, A\& $A$ 362, 1093

Rengarajan, T. N., \& Ho, P. T. P. 1996, ApJ 465, 363

Zhang, Q., et al. 2006, astroph-12027 